> Le traitement depuis 1996 de la leucémie aiguë promyélocytaire par le trioxyde d'arsenic $\left(\mathrm{As}_{2} \mathrm{O}_{3}\right)$ a ouvert la voie de son utilisation dans d'autres cancers. Fait nouveau, l'As $\mathrm{O}_{3}$ permet aussi de traiter les syndromes auto-immuns et lymphoprolifératifs de la souris MRL/lpr en induisant l'apoptose des lymphocytes $T$ activés responsables des atteintes cutanées, pulmonaires et rénales, et de la lymphoprolifération. $\mathrm{L}^{\prime} \mathrm{As}_{2} \mathrm{O}_{3}$ normalise les taux sériques de nombreuses cytokines (TNF- $\alpha$, IL-18, IFN- $\gamma$, IL-10), de Fas ligand, et des métabolites du monoxyde d'azote. En inhibant la production d'auto-anticorps, il bloque le développement de la glomérulonéphrite, ce qui conduit à une augmentation spectaculaire de la durée de vie de la souris. <

\section{De nouvelles perspectives thérapeutiques pour l'arsenic}

De la leucémie aiguë promyélocytaire aux maladies auto-immunes

Pierre Bobé, Mounira K. Chelbi-Alix

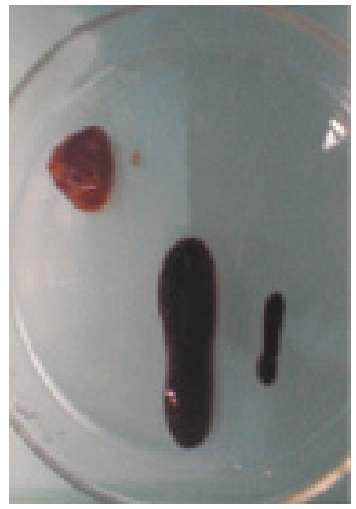

CNRS FRE 2937, Institut André Lwoff, 7 rue Guy Moquet, 94801 Villejuif Cedex, France. bobe@infobiogen.fr mchelbi@vjf.cnrs.fr
Dès l'Antiquité et jusqu'au début du XXe siècle, l'arsenic (As) a été utilisé dans le traitement d'un grand nombre de maladies. Son utilisation a été abandonnée avec la découverte des rayons $X$ et de la chimiothérapie. Dans les années 1990, son action thérapeutique a été reconsidérée sous la forme de trioxyde d'arsenic, $\mathrm{As}_{2} \mathrm{O}_{3}$, pour le traitement de la leucémie aiguë promyélocytaire (LAP), un type rare de leucémie dû à une translocation chromosomique qui engendre la synthèse de la protéine chimérique PML/RAR $\alpha$ (retinoic acid receptor $\alpha$ ) [1]. L'arsenic induit la dégradation de l'oncoprotéine PML/RAR $\alpha$ et l'apoptose des cellules leucémiques, et par conséquent la rémission de la LAP. L'efficacité thérapeutique de l'As $\mathrm{O}_{3}$ dans des hémopathies malignes et des tumeurs solides est actuellement évaluée dans plus de 40 essais cliniques [2]. Pour la première fois, nous venons de montrer que l'As $\mathrm{O}_{3}$ est également efficace pour le traitement de pathologies auto-immunes systémiques. $\mathrm{L}^{\prime} \mathrm{As}_{2} \mathrm{O}_{3}$ permet à la fois de guérir et de prévenir le développement du lupus érythémateux disséminé (LED), du syndrome de Sjögren - caractérisé par la destruction des glandes lacrymales et salivaires -, de la polyarthrite rhumatoïde (PR) et du syndrome lymphoprolifératif de la souris MRL/lpr. Ces maladies sont dues à l'accumulation, dans les organes lymphoïdes secondaires, de lymphocytes T activés à la suite d'une inactivation du récepteur d'apoptose Fas, responsable du maintien de l'homéostasie des lymphocytes $T$ en fin de réponse immunitaire. Cette revue rend compte de ces données nouvelles.

\section{Effets généraux de l'arsenic de l’Antiquité à nos jours}

L'arsenic est présent dans la nature sous sa forme minérale native ou sous forme d'As inorganique lorsqu'il est combiné au soufre, au fer, au cuivre ou encore à l'oxygène, formant alors l' $\mathrm{As}_{2} \mathrm{O}_{3}$ (anhydride arsénieux, oxyde arsénieux ou arsénite), qui est la forme utilisée en thérapeutique. L'As inorganique existe sous différentes valences $(-3,0,+3,+5)$ d'où une grande variété de composés arsenicaux aux caractéristiques chimiques différentes. Les formes dominantes sont l'arsénite $\left(\mathrm{As}_{2} \mathrm{O}_{3}\right)$ et l'arséniate $\left(\mathrm{As}_{2} \mathrm{O}_{5}\right)$ de valence respectivement III et V. De I'Antiquité Gréco-romaine jusqu'au XVIII siècle, l'As a été utilisé pour soigner toutes sortes de maux, comme des affections cutanées ou nerveuses, des tumeurs, des maladies du sang ou encore la lèpre et la peste. Durant le XIXe siècle et le début du XX' sous forme de dérivé organométallique (solution de 
Fowler, arsphénamine) a été prescrit pour traiter l'anémie, l'asthme, le psoriasis ou la syphilis $[3,4]$. $\mathrm{L}^{\prime} \mathrm{As}_{2} \mathrm{O}_{3}$ a été utilisé jusque dans les années 1930 pour le traitement des leucémies myéloïdes chroniques [5]. La découverte des traitements par rayons $X$, des antibiotiques et de la chimiothérapie a conduit à son abandon. Dans les années 1990, l'intérêt thérapeutique de l'As $\mathrm{O}_{3}$ a été reconsidéré par l'équipe de Zhu Chen à I'Institut d'hématologie de Shanghaï pour le traitement de la LAP chez des malades ne répondant plus à l'action thérapeutique de l'acide rétinoïque (AR) [1].

\section{Thérapeutique ciblée}

\section{de la leucémie aiguë promyélocytaire par l'As $\mathrm{O}_{2}$}

La LAP, qui représente $10 \%$ à $15 \%$ des leucémies aiguës myéloïdes (LAM), se caractérise par un blocage de la voie de la granulopoïèse au stade promyélocyte et par l'accumulation de promyélocytes leucémiques dans la moelle osseuse et la circulation sanguine (Figure 1). Au niveau moléculaire, la LAP se caractérise par la translocation chromosomique $\mathrm{t}(15 ; 17)$ (q22; q21) qui crée une fusion des gènes $P M L$ (promyelocytic leukemia) et $R A R \alpha$ aboutissant à la synthèse de l'oncoprotéine PML/RAR $\alpha$ [6]. Cette protéine chimère se comporte comme un dominant négatif et exerce ses effets transformants en interférant avec la fonction des récepteurs nucléaires, ce qui conduit à un blocage de la différenciation cellulaire [7]. PML/RAR $\alpha$ délocalise PML d'une structure intranucléaire multiprotéique, dénommée «corps nucléaires $P M L$ », ce qui aboutit à une dérégulation de la croissance cellulaire et de l'apoptose [8].

Des rémissions complètes de patients atteints de LAP sont obtenues depuis 1988 après administration d'AR qui restaure la différenciation des cellules leucémiques en polynucléaires neutrophiles [9]. Malgré son efficacité, certains patients développent une résistance à l'AR qui a pu être surmontée grâce à la découverte des effets thérapeutiques de l'As $\mathrm{O}_{3}[1,10,27]$.

\section{L'As ${ }_{2} \mathrm{O}_{3}$ cible spécifiquement l'oncoprotéine PML/RAR $\alpha$}

Les souris transgéniques PML/RAR $\alpha$ développent une LAP très proche de la maladie humaine [11]. Comme dans cette dernière, l'évolution de la maladie chez la souris est très rapide et conduit à la mort des animaux 4 semaines après l'apparition de cellules leucémiques dans le sang périphérique. Dans tous les cas, les leucémies développées chez

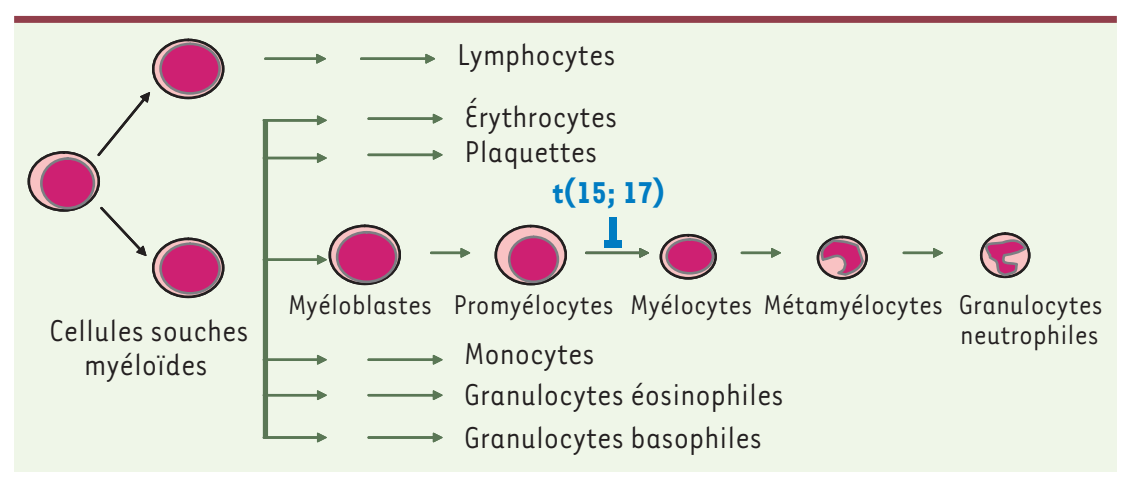

les animaux transgéniques sont transplantables à des souris immunocompétentes qui développent à leur tour une leucémie aiguë ayant les mêmes caractéristiques. $\mathrm{PML/RAR} \alpha$ bloque la différenciation cellulaire en séquestrant le corépresseur transcriptionnel SMRT/ $\mathrm{N} c o R$ (Silencing mediator of retinoid and thyroid hormone receptors/Nuclear hormone receptor corepressor) et l'histone déacétylase HDAC3. L'As $\mathrm{O}_{3}$ induit la phosphorylation de SMRT/NcoR par la voie MAPK, aboutissant à la dissociation de SMRT/NcoR de PML/RAR $\alpha$ et à la différenciation cellulaire [2]. De plus, $\mathrm{I}^{\prime} \mathrm{As}_{2} \mathrm{O}_{3}$ et I'AR induisent la dégradation par le protéasome de $P M L / R A R \alpha$ en ciblant respectivement PML et RAR $\alpha$, ce qui permet à nouveau la formation des corps nucléaires PML $[12,13]$. Ainsi, PML et RAR $\alpha$ retrouvent leur fonction entraînant la reprise des fonctions normales de la cellule (Figure 2). Cela constitue le premier modèle thérapeutique ciblant un oncogène [8].

\section{Effet de l'As $\mathrm{O}_{3}$ sur le cycle cellulaire et l'apoptose} Le cycle cellulaire est contrôlé par les cyclines dépendantes des kinases (CDK). L'exposition de nombreuses lignées tumorales à l' $\mathrm{As}_{2} \mathrm{O}_{3}$ induit l'arrêt en phase $\mathrm{Gl}$ et/ou G2-M du cycle cellulaire. Selon le type cellulaire, l'arrêt en phase Gl ou G2-M est associé ou non à une régulation négative de $\operatorname{CDKl}$ (appelée aussi kinase cdc2), CDK2, CDK6, cycline D1, cycline $\varepsilon$ et cycline $A$. L'induction de l'apoptose dans la LAP est associée à la production d'un taux important d'espèces réactives de l'oxygène (ROS). Les ROS peuvent induire l'apoptose via l'activation de la kinase Jun (JNK) [2]. Cette apoptose implique également la chute du potentiel mitochondrial suivie de l'activation de la caspase 3 [14].

\section{Enjeux thérapeutiques de l'As $\mathrm{O}_{3}$}

Dans les années 2000-2002, la Food and Drug Administration et la Commission Européenne ont autorisé la commercialisation d'As $\mathrm{O}_{3}$ (sous le nom de Trisenox) pour traiter les patients atteints de LAP récurrente ou réfractaire au traitement par I'AR. Le Trisenox est actuellement évalué dans plus de 40 études cliniques, pour son efficacité dans le traitement d'autres

Figure 1. Caractéristiques de la leucémie aiguë promyélocytaire. La LAP est caractérisée par la translocation chromosomique $\mathrm{t}(15 ; 17)$ qui fusionne les gènes $P M L$ et $R A R \alpha$ aboutissant à la synthèse de l'oncoprotéine PML-RAR $\alpha$ qui bloque les cellules au stade de promyélocyte. 


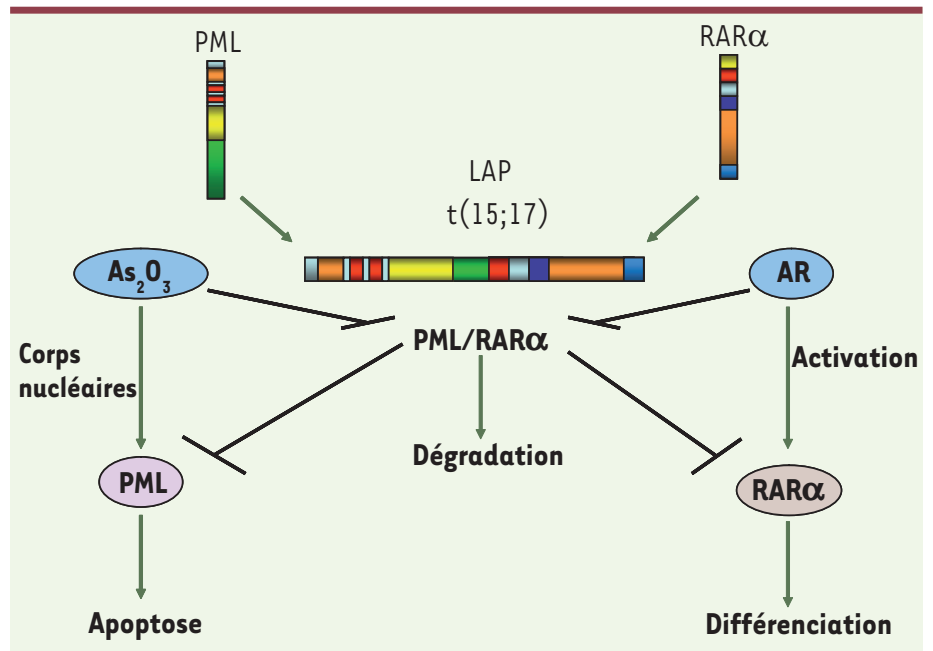

Figure 2. Mécanismes d'action de l'As203 et de l'AR dans la LAP. L'AR et l'As ${ }_{2} \mathrm{O}_{3}$ permettent la rémission des patients atteints de LAP en induisant respectivement la différenciation et l'apoptose. Les deux agents entraînent aussi la dégradation de PML/RAR $\alpha$.

\section{Le modèle de la souris MRL/Lpr}

La souris MRL/lpr est un modèle spontané de LED, de polyarthrite rhumatoïde (PR) et du syndrome de Sjögren. Les souris MRL/lpr présentent également une hyperplasie de la rate et des ganglions lymphatiques due à l'inactivation du récepteur d'apoptose Fas (mutation Ipr, pour lymphoproliferation) normalement responsable de l'élimination des lymphocytes T activés en fin de réponse immunitaire (Figures 3 et 4). Chez les patients ALPS (autoimmune tumeurs malignes dont le myélome multiple, les syndromes myélodysplasiques, les lymphomes non hodgkiniens, les leucémies aiguës et chroniques, les mélanomes, les glioblastomes et les cancers du foie et de la prostate. Les maladies auto-immunes sont la troisième cause de morbidité dans les pays développés, après les maladies cardiovasculaires et les cancers, et plus de quarante formes ont été décrites. Les médicaments les plus utilisés dans ces maladies sont les glucocorticoïdes, le méthotrexate et la ciclosporine. Cependant, ces traitements s'accompagnent d'effets secondaires importants liés à leur action immunosuppressive. Une situation que pourrait bouleverser l'utilisation d' $\mathrm{As}_{2} \mathrm{O}_{3}$.

\section{Thérapeutiques des maladies auto-immunes par l'As $\mathrm{O}_{3}$}

Un dérèglement des voies d'apoptose est incriminé dans la physiopathologie de nombreuses maladies, y compris certaines maladies auto-immunes. Grâce au modèle de la souris MRL ${ }^{\text {pr/lpr }}$ (MRL/lpr), les relations existant entre des défauts dans les voies d'apoptose responsables du maintien de l'homéostasie du système immunitaire et le développement de pathologies auto-immunes systémiques ont pu être mises en évidence.

Figure 3. Contrôle de l'homéostasie des lymphocytes $T$ par la voie Fas/FasL. A. Les lymphocytes $T$ qui expriment de façon constitutive à leur membrane le récepteur d'apoptose Fas exprimeront aussi son ligand FasL après activation par les cellules présentatrices d'antigènes (CPAg). L'interaction de FasL avec Fas induit la mort fratricide des lymphocytes T. B. Bien qu'exprimant FasL après activation, les lymphocytes $T$ des souris lpr ne pourront pas mourir par apoptose du fait de l'absence de récepteur Fas fonctionnel (Fas ${ }^{\text {lpr }}$ ), et ils s'accumuleront en périphérie (lymphoprolifération). lymphoproliferative syndrome), l'inactivation du récepteur Fas se traduit également par une splénomégalie et des adénopathies ainsi que par une hypergammaglobulinémie et des manifestations auto-immunes [15-17]. Chez la souris MRL/lpr, mais très rarement chez l'homme, la mutation du gène fas est présente à l'état homozygote ce qui explique les différences observées dans la sévérité des manifestations entre cette souris et les patients ALPS. Comme chez l'homme, la sévérité de la pathologie est liée au fond génétique puisque les souris C57BL/6-lpr/lpr développent des pathologies moins sévères que les souris MRL/lpr. De plus, la maladie est plus grave chez les souris femelles que chez les mâles, soulignant comme chez l'homme l'importance du contexte hormonal. Les lymphocytes T qui s'accumulent dans les organes lymphoïdes secondaires des patients ALPS et des souris MRL/lpr ont un récepteur d'antigène (TCR) $\alpha \beta$ et un phénotype double négatif (DN) $\mathrm{CD}^{-} \mathrm{CD} 8^{-}$. Cependant, ces lymphocytes $\mathrm{T}$ DN dérivent de lymphocytes $T$ activés $C D 4^{+} C D 8^{-}$ou $C D 4^{-} C D 8^{+}$qui, du fait de la mutation du récepteur Fas, ont échappé au processus d'autorégulation par apoptose (Figure 3) et perdu l'expression des molécules CD4 ou CD8. Ces lymphocytes T DN expriment également à leur membrane la molécule B220 qui est un marqueur de différenciation des lymphocytes

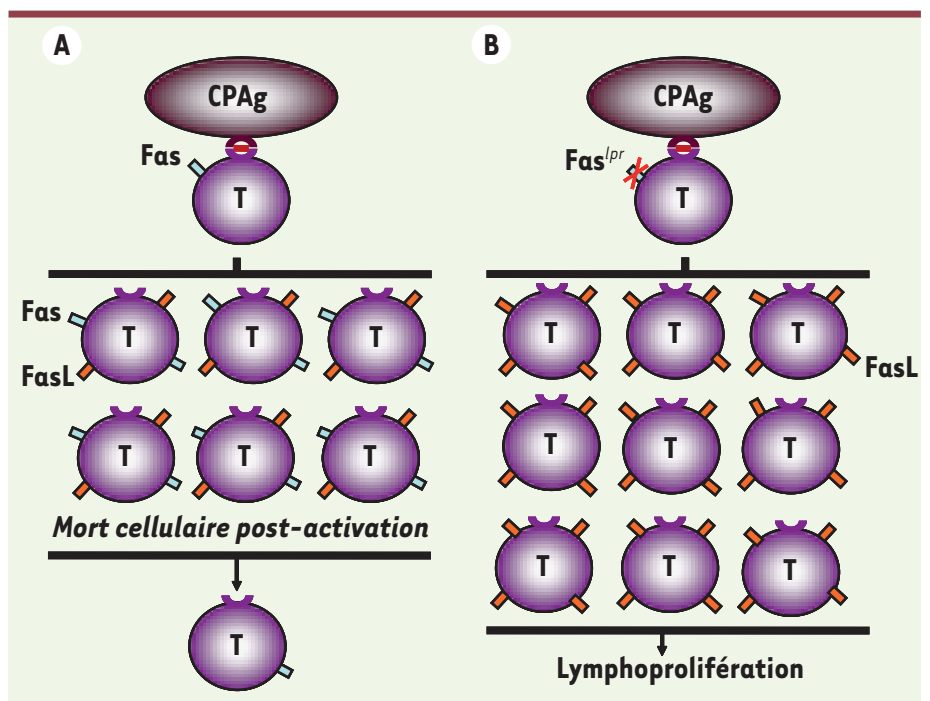




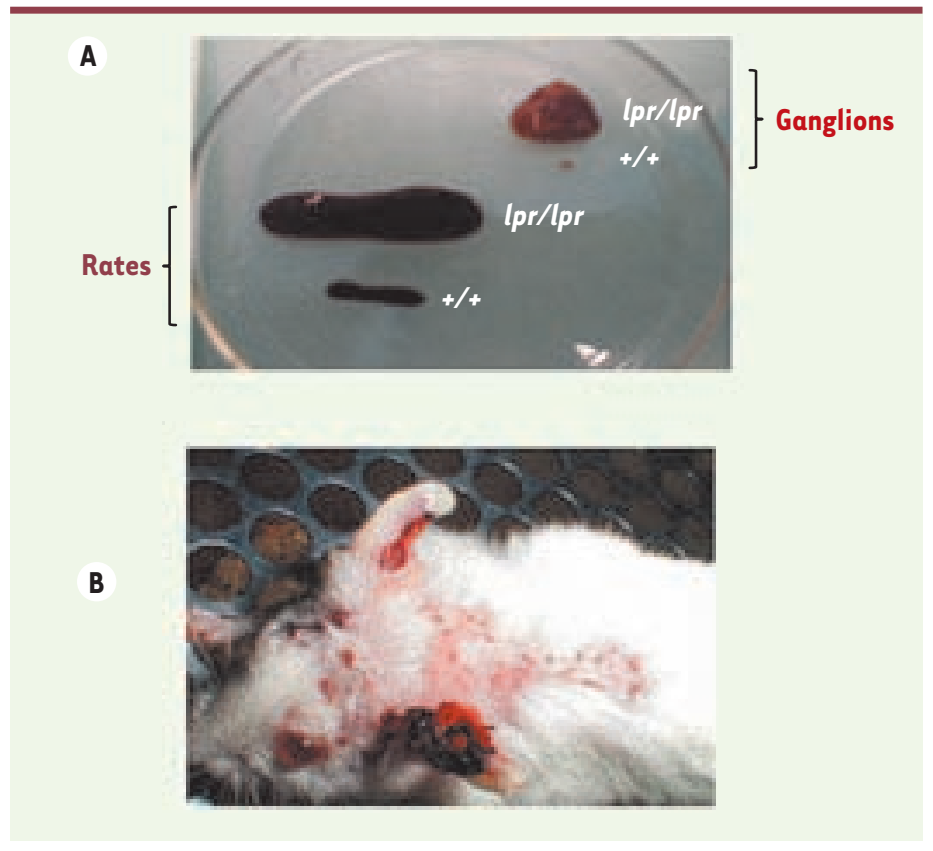

Figure 4. Prolifération lymphoïde dans les organes lymphoïdes secondaires et manifestations auto-immunes de la souris MRL/Ipr. A. Rate et ganglion de souris MRL/lpr (lpr/lpr) dont la taille est respectivement 10 et 100 fois plus importante que celle d'une souris saine $\mathrm{MRL}^{+/+}(+/+)$du fait de l'accumulation de lymphocytes $T$ activés de phénotype $\mathrm{CD}^{-}{ }^{-} \mathrm{CD} 8^{-} \mathrm{B} 220^{+} \mathrm{Fas} L^{+}$. $B$. Les atteintes cutanées et l'exophtalmie sont caractéristiques respectivement du LED et du syndrome de Sjögren chez une souris femelle MRL/lpr âgée de 4 mois.

B et des lymphocytes T activés qui ont reçu un signal d'apoptose [18]. Enfin, l'absence de récepteur Fas fonctionnel entraîne la surexpression de son ligand FasL à la membrane des lymphocytes T et B activés, les rendant spontanément cytotoxiques in vitro [19-21]. La mutation Ipr est une mutation fuyante, ce qui aboutit à l'expression d'environ $10 \%$ de protéines Fas fonctionnelles à la surface des cellules des tissus [22]. Les molécules FasL exprimées par les lymphocytes $T$ et $B$ pourraient activer les récepteurs Fas fonctionnels présents à la surface des cellules dans les tissus des souris MRL//pr, ce qui entraînerait leur destruction $[20,23]$.

\section{Rôle curatif et préventif}

\section{des pathologies auto-immunes de l' $\mathrm{As}_{2} \mathrm{O}_{3}$}

Les souris MRL/lpr ont des atteintes cutanées (Figure 4), pulmonaires, rénales et articulaires dues à des infiltrations lymphoïdes et les titres d'auto-anticorps anti-ADN et de facteur rhumatoïde (IgM, IgG ou IgA dirigées contre le fragment constant des $\lg G$ ) sont élevés. Ces auto-anticorps sont responsables de glomérulonéphrites à complexes immuns qui entraînent la mort de la souris MRL/lpr en 4 à 5 mois alors que la durée de vie moyenne d'une souris est d'environ 2 ans. La sévérité de la maladie est également liée aux taux élevés de cytokines pro-inflam-

matoires (TNF (tumor necrosis factor)- $\alpha$, IL (interleukine)-18), de FasL soluble et de cytokines de type Thl (IFN (interféron)- $\gamma$ ). De plus, les souris MRL/lpr, tout comme les patients ALPS [15], présentent un taux anormalement élevé d'IL-10 sérique, une cytokine de type Th2 impliquée dans l'inhibition de la réponse inflammatoire, mais aussi dans la différenciation des lymphocytes $\mathrm{B}$. Chez les animaux malades, le traitement par l'As $\mathrm{O}_{2} \mathrm{O}_{3}$ induit la disparition complète de la lymphoprolifération, des atteintes cutanées, pulmonaires et rénales caractéristiques du LED [24]. Fait impressionnant, les atteintes cutanées liées au LED diminuent dès le septième jour de traitement et ont disparu après 18 jours de traitement (Figure 5 ). Le traitement par l'As $\mathrm{O}_{3}$ normalise également les taux sériques de TNF- $\alpha$, FasL soluble, IL-18, IFN- $\gamma$ et IL-10 ainsi que celui des auto-anticorps. La durée de vie des souris MRL/lpr traitées par l' $\mathrm{As}_{2} \mathrm{O}_{3}$ est équivalente à celle des animaux normaux, environ 2 ans. Chez des animaux jeunes n'ayant pas encore développé la maladie, le traitement par l' $\mathrm{As}_{2} \mathrm{O}_{3}$ prévient son développement [24]. ' '’As $\mathrm{O}_{3}$ a donc un rôle aussi bien préventif que curatif dans les pathologies auto-immunes des souris MRL/lpr.

\section{Mode d'action de l' $\mathrm{As}_{2} \mathrm{O}_{3}$ chez les souris MRL/lpr}

$\mathrm{L}^{\prime} \mathrm{As}_{2} \mathrm{O}_{3}$ induit l'activation des caspases et donc l'apoptose, mais exclusivement dans les lymphocytes T anormaux DN B220+ FasL $^{+}$qui s'accumulent chez la souris MRL/lpr, alors que les lymphocytes T normaux $\mathrm{CD}^{+}$ou $\mathrm{CD} 8^{+}$ne sont pas affectés. $\mathrm{L}^{\prime} \mathrm{As}_{2} \mathrm{O}_{3}$ n'induit donc pas une immunosuppression non spécifique [24]. Par ailleurs, chez la souris MRL/lpr, on détecte une forte production de

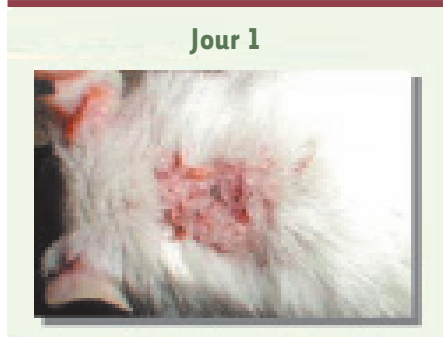

Jour 18

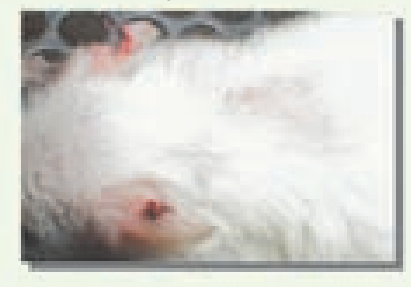

Figure 5. Traitement par l'As $\mathrm{O}_{3}$ des atteintes cutanées de type lupique des souris MRL/Ipr. Les souris MRL/lpr ont reçu 5 jours par semaine et durant 35 jours, $5 \mu \mathrm{g} / \mathrm{g}$ de poids de souris d'As $\mathrm{O}_{3}$. Évolution des atteintes cutanées caractéristiques du $L E D$ 1, 7, 18 et 35 jours après le début du traitement par $\mathrm{As}_{2} \mathrm{O}_{3}$. 
monoxyde d'azote (NO) et de ROS, en partie responsable des lésions tissulaires. Cette forte production de radicaux libres oxygénés et azotés est corrélée à un effondrement du taux de glutathion réduit (GSH) qui est le principal antioxydant cellulaire. Le traitement par l' $\mathrm{As}_{2} \mathrm{O}_{3}$ normalise les taux sériques des métabolites du NO (nitrites/nitrates) ainsi que ceux du GSH intracellulaire [24].

\section{Conclusion}

Différentes formes d'As ont été utilisées comme médicaments au cours de l'histoire de l'humanité. La découverte de la remarquable efficacité de l'As $\mathrm{O}_{3}$ dans le traitement de la LAP a conduit à évaluer son efficacité dans d'autres formes de cancers. Nos travaux récents dans le modèle de la souris MRL/lpr montrent que l'As $\mathrm{O}_{3}$ est aussi efficace pour guérir ou prévenir toutes les pathologies auto-immunes de cette souris. L'expression de cytokines comme le TNF- $\alpha$ ou l'IFN est associée à différentes maladies auto-immunes, ce qui a entraîné la mise au point et I'utilisation thérapeutique d'anticorps anti-TNF [25, 28] et anti-IFN [26]. De façon remarquable, l'As $\mathrm{O}_{3}$ normalise chez la souris auto-immune MRL/lpr la production de l'ensemble des cytokines. A la différence des approches utilisant des anticorps ou des récepteurs solubles spécifiques d'une seule cytokine, l'As $\mathrm{O}_{3}$ cible l'ensemble des cytokines dont l'expression est dérégulée. Cette découverte fait naître un espoir, celui d'utiliser l' $\mathrm{As}_{2} \mathrm{O}_{3}$ pour le traitement de pathologies auto-immunes graves chez des patients devenus réfractaires aux traitements immunosuppresseurs. Par quel(s) mécanisme(s) I' $\mathrm{As}_{2} \mathrm{O}_{3}$ cible spécifiquement les cellules leucémiques dans la LAP ou les lymphocytes $T$ responsables de la lymphoprolifération et des pathologies auto-immunes chez la souris MRL/lpr reste une question ouverte? $\diamond$

\section{SUMMARY}

New therapeutic perspectives for Arsenic:

from acute promyelocytic leukemia

\section{to autoimmune diseases}

Since 1996, arsenic trioxide $\left(\mathrm{As}_{2} \mathrm{O}_{3}\right)$ is used to treat patients with acute promyelocytic leukemia. We have recently shown that $\mathrm{As}_{2} \mathrm{O}_{3}$ is a novel promising therapeutic agent for the autoimmune diseases (human lupus-like syndrome) and the massive lymphoproliferation (human autoimmune lymphoproliferative-like syndrome) developed by $\mathrm{MRL} /$ lpr mice. $\mathrm{As}_{2} \mathrm{O}_{3}$ is able to achieve an almost complete regression of antibody- and cell-mediated manifestations in MRL/lpr mice. $\mathrm{As}_{2} \mathrm{O}_{3}$ eliminated the activated T lymphocytes responsible for lymphoproliferation and skin, lung, and kidney lesions. This treatment also markedly reduced anti-DNA autoantibodies, rheumatoid factor, IL-18, IFN- $\gamma$, nitric oxide metabolites, TNF- $\alpha$, Fas ligand and IL-10 levels, and immunecomplex deposits in glomeruli, leading to significantly prolonged survival rates. $\diamond$

\section{RÉFÉRENCES}

1. Chen GQ, Shi XG, Tang W, et al. Use of arsenic trioxide $\left(\mathrm{As}_{2} \mathrm{O}_{3}\right)$ in the treatment of acute promyelocytic leukemia (APL). I. $\mathrm{As}_{2} \mathrm{O}_{3}$ exerts dose-dependent dual effects on APL cells. Blood $1997 ; 89$ : 3345-53.

2. Dilda PJ, Hogg PJ. Arsenical-based cancer drugs. Cancer Treat Rev 2007 ; $33: 542-64$.

3. Alibert JL. Nouveaux éléments de thérapeutique et de matière médicale, suivis d'un nouvel essai sur l'art de formuler. Paris, Chez Crapart, Caille et Ravier, 2 vol 1804.

4. Lissauer H. Zwei Faelle von Leucaemie. Berl Klin Wochenschr $1865 ; 2$ : 403-5.

5. Forkner C, McNair-Scott T. Arsenic as a therapeutic agent in chronic myeloid leukemia. JAMA $1931 ; 97: 305$.

6. Lallemand-Breitenbach V, Zhu J, de Thé H. La leucémie aiguë promyélocytaire : un paradigme ciblés sur l'oncogène? Med Sci (Paris) 2001 ; 17: 14-22.

7. Grignani F, Valtieri M, Gabbianelli M, et al. PML/RARa fusion protein expression in normal human hematopoietic progenitors dictates myeloid commitment and the promyelocytic phenotype. Blood $2000 ; 96: 1531-7$.

8. De Thé H, Chelbi-Alix MK. APL, a model disease for cancer therapies? Oncogene 2001 ; $20: 7136-9$.

9. Huang $M E, Y_{e} Y C$, Chen $S R$, et al. Use of all-trans retinoic acid in the treatment of acute promyelocytic leukemia. Blood $1988 ; 72: 567-72$.

10. Soignet SL, Maslak P, Wang ZG, et al. Complete remission after treatment of acute promyelocytic leukemia with arsenic trioxide. $N$ Engl J Med 1998; 339 : 1341-8.

11. Brown D, Kogan $S$, Lagasse $\varepsilon$, et al. A PMLRARalpha transgene initiates murine acute promyelocytic leukemia. Proc Natl Acad Sci USA 1997 ; 94 : 2551-6.

12. Zhu J, Koken MH, Quignon F, et al. Arsenic-induced PML targeting onto nuclear bodies: implications for the treatment of acute promyelocytic leukemia. Proc Natl Acad Sci USA $1997 ; 94: 3978-83$.

13. Zhu J, Gianni M, Kopf $\varepsilon$, et al. Retinoic acid induces proteasome-dependent degradation of retinoic acid receptor a (RARa) and oncogenic RARa fusion proteins. Proc Natl Acad Sci USA $1999 ; 96: 14807-12$.

14. Zhang TD, Chen GQ, Wang ZG, et al. Arsenic trioxide, a therapeutic agent for APL. Oncogene $2001 ; 20: 7146-53$.

15. Rieux-Laucat F, Le Deist F, Hivroz C, et al. Mutations in Fas associated with human lymphoproliferative syndrome and autoimmunity. Science $1995 ; 268: 1347-9$.

16. Bidère N, Su HC, Lenardo MJ. Genetic disorders of programmed cell death in the immune system. Annu Rev Immunol 2006 ; 24 : 321-52.

17. Rieux-Laucat $F$. Le syndrome lymphoprolifératif avec auto-immunité : un défaut hérité ou acquis d'apoptose lymphocytaire. Med Sci (Paris) $2006 ; 22: 645-50$.

18. Renno T, Attinger A, Rimoldi D, et al. Expression of $B 220$ on activated T cell blasts precedes apoptosis. Eur J Immunol $1998 ; 28: 540-7$

19. Benihoud K, Bonardelle D, Bobé $P$, et al. MRL/lpr CD4-CD8- and CD8 $8^{+}$T cells, respectively, mediate Fas-dependent and perforin cytotoxic pathways. Eur J Immunol 1997 ; 27 : 415-20.

20. Bobé $P$, Bonardelle $D$, Reynès $M$, et al. Fas-mediated liver damage in $M R L$ hemopoietic chimeras undergoing Ipr-mediated graft-versus-host disease. J Immunol 1997 ; 159 : 4197-204.

21. Bonardelle D, Benihoud K, Kiger N, et al. B lymphocytes mediate Fas-dependent cytotoxicity in MRL/lpr mice. J Leukoc Biol 2005 ; 78 : 1052-9.

22. Adachi $M$, Suematsu $S$, Kondo $T$, et al. Targeted mutation in the Fas gene causes hyperplasia in peripheral lymphoid organs and liver. Nat Genet $1995 ; 11: 294-300$.

23. Bonardelle $D$, Bobé $P$, Reynès $M$, et al. Inflammatory arthropathy in MRL hematopoietic chimeras undergoing Fas mediated graft-versus-host syndrome. J Rheumatol $2001 ; 28$ : 956-61.

24. Bobé $P$, Bonardelle $D$, Benihoud $K$, et al. Arsenic trioxide: a promising novel therapeutic agent for lymphoproliferative and autoimmune syndromes in MRL/Ipr mice. Blood $2006 ; 108: 3967-75$.

25. Feldmann M, Maini RN. Anti-TNF-a therapy of rheumatoid arthritis: what have we learned? Annu Rev Immunol 2001 ; 19: 163-96.

26. Durelli L, Ricci A. Anti-interferon antibodies in multiple sclerosis. Molecular basis and their impact on clinical efficacy. Front Biosci 2004 ; 9 : 2192-204.

27. Parisotto M, Brodeur H, Bhat PV, Mader S. Métabolisme des rétinoïdes et cancer. Med Sci (Paris) $2006 ; 22: 1101-6$.

28. Bensussan A, Bizzini B, Pouletty P, Gallo RC, Zagury D. Les kinoïdes : Une nouvelle génération de vaccins thérapeutiques. Med Sci (Paris) 2008 ; 24 : 306-13. 